\title{
The Relationship between Substance Use, Strain, and Depression among College Students
}

\author{
Taylor Walker ${ }^{1}$ and Thomas McNulty ${ }^{1}$ \\ ${ }^{1}$ University of Georgia, Athens, GA, USA \\ DOI: https://doi.org/10.47611/jsr.v10i1.1132
}

\section{$\underline{\text { ABSTRACT }}$}

This project examined the relationship between substance use and depression using General Strain Theory as a theoretical foundation. The relationship between strain, depression, and substance use has yet to be rigorously established in the literature. The goal of this project was to determine whether depression serves as a mediator between the sociological concept of strain and substance use. The hypothesis for this project was that strain would have a positive effect upon depression and substance use, and depression will act as a mediator between strain and substance use. 832 college students between the ages of 18-25 filled out an anonymous online survey containing questions from the Beck Depression Inventory, the Drug Use Screening Inventory Revised, and the Drug Abuse Screening Test. Demographic data was also collected and served as the control variables for the analysis. Data analysis was performed using the Statistical Package for the Social Sciences version 25. Pearson correlation tests and multiple linear regression analyses were carried out on explanatory and control variables. The statistical findings of this project signify that depression acted as a mediator between strain and substance use. Comparison of findings to past studies and avenues of future research will be discussed.

\section{Introduction}

Throughout the past decade, an influx of opioids has flooded onto America's streets. Due to this, much attention has been given to the effects this epidemic has had upon the adult population. Since 2000, opiate overdose deaths have increased by 200\% (Ostling, Davidson, Anyama, Helander, Wyche, \& Kaye, 2018). Additionally, prescription rates for opioids have tripled since 1999 (Ostling et al., 2018). This signifies that America is currently undergoing a severe opioid epidemic.

It is currently estimated that 2.4 million Americans meet the criteria for severe opioid use disorder (Vashishtha, Mittal, \& Werb, 2017). Despite increased public attention and funding given to treatment programs, this epidemic does not seem to be slowing down. One group of scholars argued that this is due to the lack of medicallyassisted treatments available to offenders and the overly punitive laws America has towards drug offenders (Vashishtha, Mittal, \& Werb, 2017). Other researchers argue that it is the stigma associated with drug use and addiction that have helped perpetuate this epidemic (Buchman, Leece, \& Orkin, 2017). Furthermore, although there are databases which keep track of prescribing information for opioids, the information policymakers have available to them so that they construct effective legislation is limited due to the fact that much of the information gathered by these databases is private (Maxwell, 2011). Unfortunately, this epidemic has led to a large number of deaths. In 2017, 47,600 Americans died as a result of an opiate-involved overdose (NIDA, 2019).

There is emerging evidence to suggest that the opioid epidemic may actually be a polydrug epidemic. In 2009, it was estimated that over 7 million Americans used a prescription drug for non-medicinal purposes (Schreiner, 2012). Moreover, along with the increase in the number of prescription opiates given out, the number of prescriptions for benzodiazepines (anxiety-relievers) increased by 67\% from 1996 to 2013 (Limandri, 2018). Combined with the 
exponential increase in opiate prescriptions, this increase has helped exacerbate the number of opiate overdoses. This is due to the fact that when taken concurrently opioids and benzodiazepines can cause respiratory depression and death (Limandri, 2018). According to the National Institute on Drug Abuse (2019), 11,537 Americans died as a result of a benzodiazepine involved overdose in 2017. The literature is unclear as to why benzodiazepine prescriptions increased as opposed to other drugs, but benzodiazepines are commonly used to treat insomnia and anxiety.

There is no single systemic issue or event that precipitated this epidemic. Rather, this epidemic was most likely the result of a confluence of systemic issues and social trends. Issues in America's medical system include the exponential increase in prescriptions given out, the commercialization and widespread marketing of addictive drugs, public misperceptions on the safety of prescription drugs, and an increase in illegal vendors of prescription drugs (Schreiner, 2012). America's legal system has also played a part in bringing about this epidemic by enforcing overly punitive drug laws and reinforcing social stigma against those with substance use disorders (Kennedy-Hendricks et al., 2017).

Concurrently, rates of depression have increased around the world for the past few years. One population especially vulnerable to developing depression-like symptoms are college students. Estimates of prevalence of depression in this population range from $13 \%$ to $58 \%$ (Zullig \& Devin, 2012). Additionally, depression often shares comorbidity with other negative internalizing and externalizing behaviors, such as substance use. College students have also faced a simultaneous rise in stress. It is generally understood that, due to poor job prospects post-graduation and increased academic rigor and strain, students are more stressed out than ever before. This is apparently due to the increase in the number of students reporting major mental health problems and the unsustainable burden placed on counseling services in higher education because of this increase in demand (Flatt, 2013). Due to the increase in the prevalence of depression and stress among college students, students may be turning to substances to cope with the negative feelings that they are experiencing. Due to the increase in depression and substance use among students and the sparse research on the comorbidity of these behaviors among this population, this paper draws on Agnew's (1992) General Strain Theory to uncover whether the effect of "strain" on rates of substance use among college students is mediated by an index of depression.

\section{General Strain Theory}

General strain theory (GST) was first postulated in 1992 by Robert Agnew as a derivation of Merton's strain theory (Agnew, 1992). While Merton's version of strain theory proposes that deviance arises from a rift between economic opportunities and achieving socially acceptable goals, GST focuses on the everyday life strains that contribute to delinquency. Strain is a force that predisposes one to deviant behavior as a way to alleviate strain (Agnew, 2001). According to Agnew's theory, strain comes from three sources: the loss of valued objects, the presence of negative stimuli, and the inability to achieve a desired goal (Paternoster \& Mazerolle, 1994). As a result of experiencing strain, negative emotions begin to crop up. These emotions have been argued to mediate the effects of strain on antisocial outcomes, such as drug use, and can include anger, depression, and anxiety (Jang \& Johnson, 2003). Naturally, according to Agnew, it is these negative emotions that lead to crime. Substance use, according to general strain theory, is seen as a way of relieving or coping with these negative emotions (Agnew, 2001). Agnew (2001) suggests that strain will most likely lead to deviance when the stressors are perceived as unjust, are high in magnitude, are associated with low social control, and pressure individuals to engage in criminal behavior.

The relationship between strain and substance use has yet to be rigorously established in the literature. One study of a group of Turkish undergraduate students failed to find a significant relationship between strain and substance use while using financial and academic strain to measure strain (Özbay, 2014). However, as one group of researchers found, there are an overabundance of articles which have found significant relationships between variables associated with strain and substance use (Peck, Childs, Jennings, \& Brady, 2018). Therefore, there appears to be a well-established foundation for strain and its relation to substance use. 


\section{Depression among College Students}

Depression has become a rising concern among mental health professionals within the past decade. In addition to the general populace, many social scientists and social commentators have argued that college students are also currently going through a mental health crisis. This is supported by the fact that more students are seeking out counseling services on campus compared to previous decades, and by the fact that rates of depression and suicide have increased throughout the past few years (Flatt, 2013; Walters, Bulmer, Troiano, Obiaka, \& Bonhomme, 2018). It is estimated that depression may affect as much as $15-50 \%$ of the college student population (Zullig \& Devin, 2012; Unwin, Goodie, Reamy, \& Quinlan, 2013; Furr, Westefeld, McConnell, \& Jenkins, 2001). Certainly, this lends credence to the fact that younger generations are experiencing higher levels of negative affect.

There are multiple factors and life events that could lead to depression among students. Commonly cited stressors are predominantly individual factors that include trouble with time management, rigorous academic work, and trouble transitioning from an adolescent to an independent young adult (Deroma, Leach, \& Leverett, 2009). Additionally, other individual factors influencing the development of depression could be related to racial/ethnic discrimination and socioeconomic status (Jang \& Johnson, 2003). Other scholars have added that economic and societal trends can also play a part in a student's risk of developing a depressed mood. As one study found, students' perceptions of economic stress increased as they neared graduation, and, when there is high unemployment and an unstable economy, the prevalence of negative externalizing and internalizing behaviors increases among students, including depression (Guo, Wang, Johnson, \& Diaz, 2011).

The relationship between strain and depression has been rigorously established in the literature. It is commonly found that those who have experienced stressors are at a greater risk of experiencing depression (Peck et al., 2018). This is especially true of females as they are more likely than males to respond to strain through internalizing behaviors (depression) as opposed to externalizing behaviors (substance use) (Peck et al., 2018). However, there is mixed evidence for depression being a mediator of the effects of strain on substance use. One study found little support for depression being a mediator for strain and substance use (Peck et al., 2018). The researchers argued that depression as a mediator may be confounded due to variations between male and female responses to strain (Peck et al., 2018). More specifically, males respond to strain through externalizing behaviors while females respond to strain through internalizing behaviors. Due to this dearth in the literature, more research is needed on gender variations in response to strain and possible mediating variables concerning the relationship between strain and externalizing/internalizing behaviors.

\section{Substance Use among College Students}

It is widely understood that college is a time for expanding one's boundaries and taking on new experiences. Due to this perception, college life is stereotypically associated with experimentation with various substances. Although not necessarily due to this perception, substance use is a widely studied topic among this population. Unsurprisingly, the most commonly used drug among college students is alcohol (Walters, et al., 2018). Cannabis is the second commonly most used drug followed by tobacco (Walter et al., 2018). In 2013, the prevalence rate of illicit substance use among young adults ages 18 to 25 was $21.5 \%$ (SAMHSA, 2014). According to the Substance Abuse and Mental Health Services Administration (2014), when compared to younger and older age groups, young adults ages 18 to 25 report higher rates of substance use disorder. However, despite the current prescription drug epidemic in America, very few studies have been conducted concerning the effects this epidemic has had on this population.

\section{Current Study}


Given that there are a multitude of psychological, social, and economic variables that can influence whether one experiences depression or misuses a substance, this study will use GST as the theoretical basis for this project. Only recently have researchers conducted studies specifically on college populations concerning the relationship between substance use and depression. Additionally, very few of these studies have used GST as their theoretical basis. Due to this, there is a large gap in the literature. This study seeks to expand the literature by examining whether the link between strain and substance use is mediated by depression. The theory underlying this project is that due to the increasing rates of depression and strain among college students, students may be using drugs to cope with depression that results from strain. Additionally, due to the prevalence of opioids in America, we predict that, to cope with depression and strain, opioid use will be significantly positively correlated with depression and strain. The hypotheses for this project are outlined below.

H1. There will be a positive effect of strain on drug use.

H2. Strain will have a strong, positive effect on depression

H3. The effect of strain on substance use will be mediated by depression

H4. There will be a significant, positive relationship between opiate use and depression and strain.

These hypotheses are derived from GST. As Agnew postulated, as one experiences more strain then they are more likely to experience negative internalizing and externalizing behaviors which in the case of this study are depression and substance use (Agnew, 1992). If students are experiencing strain then they may be likely to use substances as a means of coping with depression.

\section{Data and Methods}

\section{Sample}

The sample for this study consisted of 832 students between the ages of 18-25 from a large Southeastern university. After receiving IRB approval, a list of students' emails was obtained from the university registrar. An email was sent out to students containing information on this project and a link to the consent form and survey. Additionally, a reminder email was sent out to all students two weeks after initial contact. Data collection took place over the course of one month, from the beginning to the end of October. Due to the questions asked in this study, data collection was anonymous, and participants were not given any incentive for participating in this project.

Data was collected via an online survey software, Qualtrics. The survey measured levels of depression participants had experienced within the past 12 months, types of drugs used within the past 12 months, the most used drug of a participant's lifetime, and the effects drug use has had on a participant's life. Lastly, demographic information such as age, sex, race, class year, and SES were collected at the end of the survey.

\section{Theoretical Measures}

Substance use was measured using part of the Drug Use Screening Inventory Revised (DUSI-R). Participants were asked how many times per month they had used a drug within the past 12 months. Answers were coded on a scale of 0-3 with 0 representing using not using a drug at all and 3 representing using a drug more than $10+$ times a month. The types of drugs listed included alcohol, marijuana, amphetamines, tobacco, prescription opiates, and illegal opiates. Participants were given an example of each drug, such as oxycodone for prescription opiates. Participants' answers to these questions were summed together and formed a substance use scale ranging from 0-23. Lastly, participants were asked which drug out of those listed, barring alcohol, they had used the most within their lifetime. 
The effects of and problems related to substance use were measured using the Drug Abuse Screening Test (DAST). The DAST is a 25-question test that asks participants yes or no questions concerning problem behaviors related to or due to drug use. Example questions include "Have you abused prescription drugs" and "Have you gotten into fights when under the influence of a drug". Answers were coded as 0 and 1 with $0=$ no and $1=y e s$. Answers to these questions were summed together and formed a substance use problems scale ranging from 0-16. For this study, strain was operationalized as stress level. At the end of this test, participants were asked to self-report their overall stress level within the past 12 months. Answers were arranged on a 5-point interval scale ranging from "very stressed" to "not stressed at all".

Depression was measured using questions from the Beck Depression Inventory (BDI). Participants answered 21 questions concerning their experiences of thoughts and feelings related to depression. Participants were presented with four answer choices for each question indicating the severity of those thoughts or feelings. Each answer was rated on a scale of 0-3 with higher values representing thoughts and feelings associated with depression. Depression was measured on a scale of 0-54 according to the guidelines set by the BDI. Participants who scored between the range of 0-16 were rated as having no or minimal depression while participants who scored between the range of 17-30 were rated as having moderate depression. Participants who had a score of $31+$ were rated as having severe depression. This scoring was performed only for descriptive purposes and was not used in the analysis.

\section{Control Variables}

Control variables for this study consisted mainly of demographic variables. More specifically, information regarding biological sex, age, SES, race/ethnicity, and class year was collected at the end of the survey. Biological sex was coded on a scale of 0-1 with 0 being coded as female and 1 being coded as male. SES was coded on a scale of 0-3 with 0 being classified as lower class, 1 was classified as lower middle class, 2 was classified as upper middle class, and 3 was classified as upper class. SES was a self-report variable that was dependent on the respondent's perception of their parents' SES. Race/ethnicity was coded on a scale of $0-1$ with 0 being White and 1 being non-White. Class year was coded on a scale of 0-3 with 0 being classified as a first year, 1 being classified as a second year, 2 being classified as a third year, and 3 being classified as students in their fourth year of college or beyond.

\section{Analytic Strategy}

Data analysis was performed using the Statistical Package for the Social Sciences (SPSS) version 25. Pearson correlation tests were performed on the variables of strain, depression, and substance use. Specifically, strain was compared to level of depression and level of depression was compared to substance use and substance use problems. A linear regression analysis was performed to illustrate the effect of strain on depression, the hypothesized mediator. Another linear regression analysis was performed using substance use as the dependent variable and demographic variables, stress, and depression as the independent variables. The goal of this regression was to see whether depression could explain away the effect of strain.

\section{Results}

\section{Descriptive Statistics}

Descriptive statistics for variables for this project are shown in Table 1 . Approximately $70 \%$ of the sample was made up of females (Table 1). As Table 1 shows, the most common race/ethnicity among respondents was White (75\%) followed by Asian-American (9.3\%). The socio-economic status of most participants was upper middle class (56.2\%) followed by lower middle class (31.3\%). It should be noted that in the analysis below only two categories existed for 
the race variable, whites (75\%) and non-whites (25\%). Upper class and lower class participants made up only 5.1\% and $7.4 \%$ of the sample respectively (Table 1). According to Table 1, most participants were in their 3rd year of college $(24.4 \%)$ followed by those in their 4 th year of college $(22.4 \%)$. The age range for this study was $18-26$ years old. The average age of a participant was 20.7 years old. These variables constituted the control variables for this study.

Table 1 also shows the results of test variables. Concerning the most important variable in this project, stress, a majority of participants (53.3\%) reported that they were stressed for the past year. This is compared to only $10.8 \%$ of participants who reported that they were not stressed for the past year. In regard to the depression variable, most participants (56.8\%) reported that they had experienced no/minimal depression within the past year. Only $13.0 \%$ of participants met the score for severe depression. Very few participants used prescription opiates (3.2\%) within the past year. Additionally, almost no participants (.03\%) met the score on the DAST for a substance use problem.

Table 1. Table of Descriptive Statistics

\begin{tabular}{|c|c|c|c|}
\hline & & $\mathrm{N}$ & $\%$ \\
\hline \multicolumn{4}{|l|}{ Biological Sex } \\
\hline & Male & 178 & $30.0 \%$ \\
\hline & Female & 416 & $70.0 \%$ \\
\hline & Total & 594 & \\
\hline \multicolumn{4}{|l|}{ Race } \\
\hline & White & 445 & $75.0 \%$ \\
\hline & $\begin{array}{c}\text { African- Ameri- } \\
\text { can }\end{array}$ & 46 & $7.8 \%$ \\
\hline & Hispanic & 32 & $5.4 \%$ \\
\hline & Asian-American & 55 & $9.3 \%$ \\
\hline & Other & & \\
\hline & & 15 & $2.5 \%$ \\
\hline & Total & & \\
\hline & & 593 & \\
\hline \multicolumn{4}{|l|}{ Class Year } \\
\hline & $1^{\text {st }}$ & 97 & $16.5 \%$ \\
\hline & $2^{\text {nd }}$ & 109 & $18.5 \%$ \\
\hline & $3^{\text {rd }}$ & 144 & $24.4 \%$ \\
\hline & $4^{\text {th }}$ & 132 & $22.4 \%$ \\
\hline & $5^{\text {th }}+$ & 107 & $18.2 \%$ \\
\hline & Total & 589 & \\
\hline \multicolumn{4}{|l|}{ SES } \\
\hline & Lower Class & 44 & $7.4 \%$ \\
\hline & $\begin{array}{c}\text { Lower Middle } \\
\text { Class }\end{array}$ & 186 & $31.3 \%$ \\
\hline & $\begin{array}{l}\text { Upper Middle } \\
\text { Class }\end{array}$ & 334 & $56.2 \%$ \\
\hline & Upper Class & 30 & $5.1 \%$ \\
\hline & Total & 594 & \\
\hline
\end{tabular}


Table 2 shows the Pearson Correlations among test variables. Significant findings are that both depression and strain are significantly positively correlated $(\mathrm{p}<.01, \mathrm{r}=.476)$. Depression $(\mathrm{p}<.01, \mathrm{r}=.190)$ and strain $(\mathrm{p}<.05, \mathrm{r}=.086)$ were also significantly positively correlated with substance use. Depression $(\mathrm{p}<.01, \mathrm{r}=.111)$ and strain $(\mathrm{p}<.05, \mathrm{r}=.088)$ were also significantly positively correlated with prescription opiate use. These relationships signify that most of the variables tested in this study are significantly and positively related to one another.

Table 2. Pearson Correlations among test variables

\begin{tabular}{|c|c|c|c|c|c|}
\hline & Drug Use & Depression & Stress & $\begin{array}{c}\text { Drug Prob- } \\
\text { lems }\end{array}$ & $\begin{array}{c}\text { Prescription } \\
\text { Opiate Use }\end{array}$ \\
\hline Drug Use & 1 & & & & \\
\hline Depression & $.190 * *$ & 1 & & & \\
\hline Stress & $.086^{*}$ & $.476^{* *}$ & 1 & & \\
\hline Drug Problems & $.488 * *$ & $.222 * *$ & .025 & 1 & \\
\hline Prescription Opiate Use & $.239 * *$ & $.111^{* *}$ & $.088 *$ & $.107 * *$ & 1 \\
\hline
\end{tabular}

Note. **. Correlation is significant at the 0.01 level (2-tailed). *. Correlation is significant at the 0.05 level (2-tailed).

\section{Multivariate Analysis}

Table 3 shows the linear regression analysis using depression as the dependent variable. This analysis was divided into three models. Model 1 included only demographic variables. Out of these variables, SES $(\mathrm{p}<.01, \mathrm{~b}=-1.91, \mathrm{t}=\mathrm{-}$ 2.745) was the only one that had a significant effect on depression. Model 2 incorporated substance use and substance use problems variables. Both substance use problems $(\mathrm{p}<.001, \mathrm{~b}=1.077, \mathrm{t}=3.757)$ and substance use $(\mathrm{p}<.01, \mathrm{~b}=.423$, $\mathrm{t}=3.049)$ had a significant effect on depression. Lastly, Model 3 incorporated the stress variable. Stress $(\mathrm{p}<.001$, $\mathrm{b}=7.722, \mathrm{t}=12.382$ ) has a significant effect on and was highly predictive of depression. This model accounted for $27.9 \%$ of the variation in depression. When compared to the R2 of previous models, this is a significant increase. Given the large effect size stress has upon depression and the large increase in the R2, this finding also supports the idea that depression acts as a mediator between strain and substance use.

Table 3. Linear regression analysis for the relationship between dependent variable depression and explanatory variables

\begin{tabular}{cccc}
\hline & $(1)$ & $(2)$ & $(3)$ \\
\hline SES & & & \\
White & $-1.91^{* *}$ & $-2.461^{* * *}$ & $-1.683^{* *}$ \\
Age & -1.644 & -2.143 & $-2.051^{*}$ \\
Female & -.561 & -.43 & -.393 \\
Class Year & 1.274 & $2.597^{*}$ & .795 \\
Drug Use & .371 & .218 & -.027 \\
Drug Problems & & $.423^{* *}$ & $.271^{*}$ \\
Stress & & $1.077^{* * *}$ & $1.088^{* * *}$ \\
\hline
\end{tabular}

Note. Alpha $=.05 . * * *=\mathrm{p}<.001, * *=\mathrm{p}<.01, *=\mathrm{p}<.05 . \mathrm{N}=578$. The dependent variable for all regressions was depression.

Table 4 shows the linear regression analysis using substance use as the dependent variable. This analysis was divided into two models. Model 1 included demographic variables and the stress variable. Stress $(\mathrm{p}<.01, \mathrm{~b}=.638, \mathrm{t}=2.745)$, sex 
$(\mathrm{p}<.001, \mathrm{~b}=-1.559, \mathrm{t}=-4.550)$, and race $(\mathrm{p}<.01, \mathrm{~b}=1.160, \mathrm{t}=3.189)$ both had a significant effect on substance use. These effect sizes show that white males are more likely to engage in substance use and that the more stressed out one is the more likely they are to engage in substance use. This model accounted for $6.9 \%$ of the variation in substance use. Model 2 added the depression variable. Depression $(\mathrm{p}<.001, \mathrm{~b}=.072, \mathrm{t}=4.762)$ had a significant effect on substance use. This shows that as one's depression level increases the likelihood that they engage in substance use increases. This model accounted for $10.3 \%$ of the variation in substance use. It should be noted that stress had a significant effect on substance use in Model 1 but not Model 2, after the depression variable is added. This signifies that depression may be acting as a mediator between strain and substance use.

Table 4. Linear regression analysis for the relationship between dependent variable substance use and explanatory variables

\begin{tabular}{ccc}
\hline & $(1)$ & $(2)$ \\
\hline SES & & \\
White & $.561 *$ & $.648 * *$ \\
Age & $1.160 * *$ & $1.302 * * *$ \\
Female & -.124 & -.088 \\
Class Year & $-1.559 * * *$ & $-1.541 * * *$ \\
Stress & .293 & .289 \\
Depression & $.638 * *$ & .064 \\
\hline
\end{tabular}

Note. Alpha $=.05 . * * *=\mathrm{p}<.001, * *=\mathrm{p}<.01, *=\mathrm{p}<.05 . \mathrm{N}=578$. The dependent variable for all regressions was substance use.

\section{Discussion}

The aim of this study was to determine whether depression serves as a mediator for the relationship between strain and substance use by using GST as a theoretical framework. Additionally, another goal of this study was to determine whether there was a significant relationship between prescription opiate use and depression and strain based off of the ongoing opioid epidemic and rise in depression and stress levels among college students. This study hypothesized that the effect of strain on substance use will be mediated by depression, and that stress and depression will have a significant effect upon prescription opiate use. The implications of the results of this study are discussed below.

Past studies have found that depression does not seem to mediate the relationship between strain and substance use (Peck et al., 2018). Rates of stress and depression have been increasing for the past decade among college students (Flatt, 2013). Additionally, other studies have found that depression is prevalent in $15 \%$ to $50 \%$ of the college student population (Zullig \& Devin, 2012; Unwin et al., 2013; Furr et al., 2001). Depression and stress also vary by country as one study which used the BDI found (Baron \& Matsuyama, 1988). This study added to the literature by examining whether depression serves as a mediator between strain and substance use. This study also added to the literature by examining the relationship between prescription opiate use and strain.

This study found that a majority of students (53.3\%) felt stressed throughout the past year, but a majority of students $(56.8 \%)$ only experienced minimal or no depression during the past year (Table 1). These findings are similar to the ones by Baron and Matsuyama (1988) who found that depression does not necessarily come from strain. However, this study used a sample of American and Japanese students as opposed to this study which only used American students (Baron \& Matsuyama, 1988). It is also hard to determine whether these findings support the prevalence rate of depression outlined in past studies. However, $43.1 \%$ of the sample qualified for borderline clinical depression due to scoring a $17+$ on the BDI (Table 1). Therefore, it is fair to say this finding is supported by past studies. 
This study found a significant relationship between not only strain and substance use but also strain and depression. These findings support the hypothesis that strain will have a positive effect on substance use (H1) and will have a strong, positive effect on depression (H3). Support for H1 refutes the findings of past studies which found strain had no effect on substance use (Özbay, 2014). However, H1 and H3 are both supported by more recent studies (Peck et al., 2018).

As shown in Table 4, depression seems to be a mediator for strain and substance use. These findings support the hypothesis that strain and substance use are mediated via depression (H2). Due to this, this study found little support for the null hypothesis. This goes against the findings of recent studies which found that depression was not a mediator (Peck et al., 2018). However, this could be due to the different samples this study had as compared to the previous study which had a more balanced gender distribution and a lower age range (Peck et al., 2018). Additionally, this study only examined substance use over the course of one year while the previous study examined substance use over the course of several years (Peck et al., 2018). Of course, due to the lack of research in this specific area of the literature, more studies are needed before this claim could be considered rigorous. Although this study was not able to establish the causal order of these variables, it appears that strain may affect substance use through depression. Of course, this is an avenue that only future studies will be able to answer.

This study also examined the use of prescription opiates among students. Due to the ongoing opioid epidemic, we hypothesized that opioid use would be prevalent and that it would be significantly correlated with depression and strain. This study found that only 20 students $(\mathrm{N}=626)$ had used prescription opiates within the past year. However, depression and stress were significantly positively correlated with prescription opiates. This finding supports hypothesis H4. In other words, it seems that the use of prescription opiates is related to depression and strain.

In addition to the implications this study has for GST, the findings of this study may also be useful for college administrators and mental health professionals that deal with student populations. As noted earlier, college campuses are experiencing a mental health crisis (Flatt, 2013). This study has shown the need for more mental health services on college campuses due to the high percentage of students suffering from severe stress and depression. Furthermore, strain could also be a useful indicator that mental health professionals can use to help clarify the mental health crisis that students may be experiencing. However, this concept would have to be refined through future studies so that it could apply to clinical settings.

This study was limited by the small sample size of only 832 participants. Additionally, the sample was predominantly female which may have skewed the results due to common variances in stress and depression due to gender as noted in the literature (Peck et al., 2018). It is also possible that effects may vary across race/ethnic groups (P. Ratanasiripong, Burkey, \& N. Ratanasiripong, 2009). Furthermore, while the participants' backgrounds were fairly diverse, these findings may not apply to the general population due to the fact that only a college population was sampled. Future studies should certainly utilize a larger sample and survey those outside the college environment. Given that this study focused on emotional/individual stressors, other studies can also examine other sources of strain such as racial discrimination, economic stress, and academic failure. What still remains unclear in the literature is the causal order of these variables. In other words, whether strain leads to depression which leads to substance use or whether strain leads to substance use which leads to depression. Given the cross-sectional nature of the data, it is not possible to determine the causal order of these relationships. While this study is purely correlational, the findings from this project could lead future projects in the right direction.

Overall, this project found that depression may mediate the effect of strain on substance use, and few students are using prescription opiates. Future studies are needed to verify this relationship and establish a causal order. Additionally, future studies should also sample populations outside of universities to ensure that this relationship is generalizable to all populations.

\section{References}

Agnew, R. (1992). Foundation for a general strain theory of crime and delinquency. Criminology, 30(1), 47-87. 
Agnew, R. (2001), Building on the foundation of general strain theory: Specifying the types of strain most likely to lead to crime and delinquency. Journal of Research in Crime and Delinquency, 38, 319-361.

Baron, M. R., \& Matsuyama, Y. (1988). Symptoms of depression and psychological distress in United States and Japanese college students. Journal of Social Psychology, 128(6), 803-816.

Buchman, D. Z., Leece, P., \& Orkin, A. (2017). The epidemic as stigma: The bioethics of opioids. Journal of Law, Medicine \& Ethics, 45(4), 607-620.

Deroma, V. M., Leach, J. B., \& Leverett, J. P. (2009). The relationship between depression and college academic performance. College Student Journal, 43(2), 325-334.

Flatt, A. K. (2013). A suffering generation: Six factors contributing to the mental health crisis in North American higher education. College Quarterly, 16(1).

Furr, R. S., Westefeld, S. J., McConnell, N. G., \& Jenkins, M. J. (2001). Suicide and depression among college students: A decade later. Professional Psychology: Research and Practice, 32, 97-100.

Guo, Y., Wang, S., Johnson, V., \& Diaz, M. (2011). College students' stress under current economic downturn. College Student Journal, 45(3), 536-543.

Jang, B. R., \& Johnson, S. J. (2003). Strain, negative emotions, and deviant coping among african americans: A test of general strain theory. Journal of Quantitative Criminology, (1), 79-105.

Kennedy-Hendricks, A., Barry, C. L., Gollust, S. E., Ensminger, M. E., Chisolm, M. S., \& McGinty, E. E. (2017). Social stigma toward persons with prescription opioid use disorder: Associations with public support for punitive and public health-oriented policies. Psychiatric Services, 68(5), 462-469.

Limandri, B. J. (2018). Benzodiazepine use: The underbelly of the opioid epidemic. Journal of Psychosocial Nursing and Mental Health Services, 56(6), 11-15.

Maxwell, J. C. (2011). The prescription drug epidemic in the United States: A perfect storm. Drug \& Alcohol Review, 30(3), 264-270.

National Institute on Drug Abuse (2018). Overdose death rates. Retrieved from https://www.drugabuse.gov/relatedtopics/trends-statistics/overdose-death-rates

Ostling, P. S., Davidson, K. S., Anyama, B. O., Helander, E. M., Kaye, A. D., \& Wyche, M. Q. (2018). America's opioid epidemic: A comprehensive review and look into the rising crisis. Current Pain and Headache Reports, 22(5).

Özbay, Ö. (2014). General strain theory and substance use. Turkish Journal of Police Studies, 16(1), 81-102.

Paternoster, R., \& Mazerolle, P. (1994). General strain theory and delinquency: A replication and extension. Journal of Research in Crime and Delinquency, 31(3), 235-263. 
Peck, J. H., Childs, K. K., Jennings, W. G, \& Brady, C. M. (2018). General strain theory, depression, and substance use: Results from a nationally representative, longitudinal sample of white, african-american, and hispanic adolescents and young adults. Journal of Child \& Adolescent Substance Abuse, 27(1), 11-28.

Ratanasiripong, P., Burkey, H., \& Ratanasiripong, N. (2009). Stress and substance use among asian american and latino college students. College Student Journal, 43(4), 1253-1259.

Schreiner, M. D. (2012). A deadly combination: The legal response to America's prescription drug epidemic. The Journal of Legal Medicine, 33(4), 529-539.

Substance Abuse and Mental Health Services Administration. (2014). Results from the 2013 National Survey on Drug Use and Health: Summary of national findings (SAMHSA Publication No. 14-4863). Retrieved from https://www.samhsa.gov/data/sites/default/files/NSDUHresultsPDFWHTML2013/Web/NSDUHresults2013.pdf

Unwin, B. K., Goodie, J., Reamy, B. V., \& Quinlan, J. (2013). Care of the college student. American Family Physician, 88(9), 596-604.

Vashishtha, D.,Mittal, M. L., \& Werb, D., (2017). The North American opioid epidemic: Current challenges and a call for treatment as prevention. Harm Reduction Journal, 14(1), 1-6.

Walters, K. S., Bulmer, S. M., Troiano, P. F., Obiaka, U., \& Bonhomme, R., (2018). Substance use, anxiety, and depressive symptoms among college students. Journal of Child \& Adolescent Substance Abuse, 27(2), 10311.

Zullig, K. J., Divin, A.L., (2012). The association between non-medical prescription drug use, depressive symptoms, and suicidality among college students. Addictive Behaviors, 37(8), 890-899. 